\title{
Analysis of T Wave Nonlinear Dynamics for Serum Potassium Monitoring in End-Stage Renal Disease Patients
}

\author{
Sabarathinam Srinivasan ${ }^{1}$, Hassaan A Bukhari $^{1,2}$, Pablo Laguna $^{1,3}$, Carlos Sánchez $^{1,4}$, Esther Pueyo ${ }^{1,3}$ \\ ${ }^{1}$ I3A, University of Zaragoza, IIS Aragón, Zaragoza, Spain \\ ${ }^{2}$ University of Bordeaux, IMB, UMR 5251, Talence, France \\ ${ }^{3}$ CIBER en Bioingeniería, Biomateriales y Nanomedicina, Spain \\ ${ }^{4}$ Defence University Centre (CUD), AGM, Zaragoza, Spain
}

\begin{abstract}
Non-invasive estimation of serum potassium, $\left[\mathrm{K}^{+}\right]$, is of major importance to prevent associated risks, but current ambulatory estimation methods are limited. We investigated changes in $T$ wave nonlinear dynamics by quantifying a divergence-related marker $\psi$ on electrocardiograms (ECGs) from 15 end-stage renal disease (ESRD) patients undergoing hemodialysis (HD) and we assessed the relationship between $\psi$ and $\left[\mathrm{K}^{+}\right]$. ECGs from 22 simulated transmural ventricular fibers were additionally calculated. In ESRD patients, $\psi$ took the largest values at the beginning and end of the HD session, corresponding to the highest and lowest $\left[\mathrm{K}^{+}\right]$values. The median correlation coefficient over patients between the change in $\psi$ and the change in $\left[\mathrm{K}^{+}\right]$was 0.92 and decreased to 0.74 after controlling for the effects of $\left[\mathrm{Ca}^{2+}\right]$ and heart rate. These associations were, however, highly patient-dependent. Both the strength and variability of the $\psi$ - $\left[\mathrm{K}^{+}\right]$relationship was reproduced in the simulations, with the variability explained by differences in transmural heterogeneities: $10 \%$ variations in the proportion of epicardial and midmyocardial cells led to more than 10\% and $8 \%$ changes in $\psi$, respectively. In conclusion, changes in the nonlinear dynamics of the ECG $T$ waves can be related to $\left[\mathrm{K}^{+}\right]$variations in ESRD patients, despite the high inter-individual variability.
\end{abstract}

\section{Introduction}

Around $10 \%$ of the worldwide population are affected by chronic kidney disease. The economic cost, increased mortality risk and decreased quality of life associated with this disease are remarkably high [1]. At the final stage, so called end-stage renal disease (ESRD), patients present an increasingly impaired ability to maintain potassium homeostasis. Serum potassium levels $\left(\left[\mathrm{K}^{+}\right]\right)$ outside normal ranges, in the form of hypokalemia or hyperkalemia, increase the risk for life-threatening arrhythmias and sudden cardiac death [2].

Investigating the effects of $\left[\mathrm{K}^{+}\right]$variations on the electrical activity of the heart could allow deriving electrocardiogram (ECG)-based markers for continuous monitoring of $\left[\mathrm{K}^{+}\right]$, which would facilitate the delivery of more timely therapies for ESRD patients. Variations in $\left[\mathrm{K}^{+}\right]$levels have been indeed shown to affect depolarization and repolarization features of the ECG [2]. Most of the investigated ECG markers in the literature are aimed at characterizing $\mathrm{T}$ wave properties [3]. However, such properties commonly refer to a specific time point or small portion of the $\mathrm{T}$ wave and are, thus, not necessarily representative of the whole $\mathrm{T}$ wave morphology changes in response to varying $\left[\mathrm{K}^{+}\right]$.

In previous studies, we characterized changes in the $\mathrm{T}$ wave shape at varying $\left[\mathrm{K}^{+}\right]$by using time-warping techniques over clinical and simulated ECGs $[4,5]$. The linear but also the nonlinear components of the measured warping markers showed a strong relationship with $\left[\mathrm{K}^{+}\right]$. Based on those results, we hypothesized that evaluation of nonlinear dynamics of the $\mathrm{T}$ wave, as measured by other techniques quantifying the divergence of trajectories starting close to each other, could provide relevant information for $\left[\mathrm{K}^{+}\right]$estimation. We quantified a divergence-related marker $\psi$ over ECGs of ESRD patients during a hemodialysis (HD) session, where large $\left[\mathrm{K}^{+}\right]$changes are expected to occur. Also, we simulated a set of ventricular fibers covering a wide range of transmural heterogeneities and we calculated pseudo-ECGs (pECGs) at different $\left[\mathrm{K}^{+}\right]$levels. After confirming that simulated $\psi$ reproduced the evidences from clinical ECGs, we performed a sensitivity analysis to assess the contribution of transmural heterogeneities to inter-individual differences in $\mathrm{T}$ wave nonlinear dynamics, particularly for $\left[\mathrm{K}^{+}\right]$values out of normal ranges. 


\section{Methods}

\subsection{Processing of ECG signals}

48-hour 12-lead ECGs were recorded from 15 ESRD patients at Hospital Clínico Universitario de Zaragoza (HCUZ). ECG acquisition started 5 minutes before the HD treatment onset and lasted for 48 hours, as shown in Figure $1[4,5]$. Concurrently, six blood samples were taken at the HD onset, at every hour during the HD session, at the HD end (minute 215 or 245 , depending on the patient) and after 48 hours, immediately before the next HD session (Figure 1, K1 to K6). Extracellular potassium and calcium concentrations, $\left[\mathrm{K}^{+}\right]$and $\left[\mathrm{Ca}^{2+}\right]$, as well as heart rate were measured at those time points. The ethical committee approved the study protocol and all patients provided signed informed consent (CEICA ref. PI18/003).

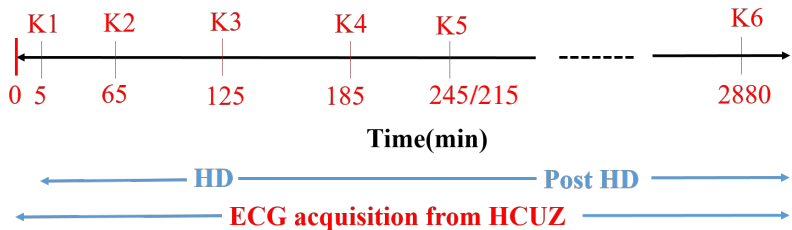

Figure 1. Diagram of the study protocol. K1 to K6 are the time points (in minutes) for blood sample extraction.

ECG pre-processing included baseline wander removal, muscle and electric noise removal as well as single-lead delineation using a wavelet-based automatic algorithm [6]. To enhance the $\mathrm{T}$ wave energy and allow more precise delineation, spatial principal components (PCs) were obtained by computing the inter-lead auto-correlation matrix of $\mathrm{T}$ waves in a stable ECG segment at the end of the HD session. The ECG recording was subsequently projected onto the direction of the 1st PC and the corresponding $\mathrm{T}$ waves were again delineated using the method described in [6] to determine the locations of their onset, peak and end.

Mean warped T waves (MWTWs), representing optimal averages in the time and amplitude domains [7], were calculated at each of the analyzed time points when blood samples were extracted. For this calculation, $\mathrm{T}$ waves in two-minute windows before the blood extraction time point were aligned, iteratively warped and averaged to compute the MWTW, as fully described in $[4,5]$.

\subsection{Computational modeling simulation}

Transmural electrical propagation from ventricular endocardium to epicardium was simulated using one-dimensional fibers of $1.65 \mathrm{~cm}$ in length. The action potential (AP) model of Ten Tusscher and Panfilov [8], with the updates by Severi et al. [9], was used to represent human ventricular cell electrophysiology. Transmural heterogeneities were simulated for a total of 22 combinations with variations in the proportions of endocardial (from $10 \%$ to $50 \%$ ), midmyocardial (from $10 \%$ to $50 \%$ ) and epicardial cells (from $20 \%$ to $80 \%$ ) [5].

A series of 10 stimuli was applied to the endocardial end of the fiber every $1000 \mathrm{~ms}$ with an amplitude equal to 1.5 times the diastolic threshold. A finite element method based software [10] was used to compute electrical propagation with a time step of $0.01 \mathrm{~ms}$ and space discretization of $0.01 \mathrm{~cm}$. pECGs were computed as in [11] and the $T$ waves were also delineated with [6].

Simulations were run for the reference physiological level in the Ten Tusscher-Panfilov model, i.e. $\left[\mathrm{K}^{+}\right]=5.4$ $\mathrm{mM}$, as well as two different levels below and above it: $\left[\mathrm{K}^{+}\right]=3,4,5.4,6.2,7 \mathrm{mM}$.

\subsection{T wave nonlinear dynamics}

The $\mathrm{T}$ wave nonlinear dynamical content was described by evaluation of a marker $\psi$ based on the divergence of trajectories starting close to each other [12]. $\psi$ was calculated for the patients' ECGs and the simulated ECGs. Let a given MWTW, upsampled by a factor 5, be denoted by $\boldsymbol{x}=\left[x_{1}, x_{2}, \ldots, x_{N}\right]$. The reconstructed trajectories for a delay $\tau$ were represented by vectors $\boldsymbol{y}_{j}=\left[x_{j}, x_{j+\tau}, \ldots, x_{j+(m-1) \tau}\right], j=1,2, \ldots, M$. Here, $M=N-(m-1) \tau$, being $m$ the embedding dimension set to 9 and $\tau$ the delay in samples set to 15 .

For each $\boldsymbol{y}_{j}$, its nearest neighbor $\boldsymbol{y}_{\hat{j}}$ was searched by minimizing:

$$
d_{j}(0)=\min _{\boldsymbol{y}_{\hat{j}}}\left\|\boldsymbol{y}_{j}-\boldsymbol{y}_{\hat{j}}\right\|,
$$

with $|j-\hat{j}|>p$, being $p$ set to $\left\lceil\frac{N}{100}\right\rceil$. The notation $\|\cdot\|$ represents the Euclidean norm.

Next, the distance between the nearest neighbors $\boldsymbol{y}_{j}$ and $\boldsymbol{y}_{\hat{j}}$ was computed after $i$ steps as:

$$
d_{j}(i)=\left\|\boldsymbol{y}_{j+i}-\boldsymbol{y}_{\hat{j}+i}\right\|,
$$

where $i=1,2, \ldots, I$, being $I$ set to $\left\lceil\frac{N}{5}\right\rceil$.

The marker $\psi$ was computed as:

$$
\psi=\frac{1}{I} \sum_{i=1}^{I} \ln (e(i)),
$$

where $e(i)=\frac{1}{M} \sum_{j=1}^{M} d_{j}(i)$.

Figure 2 illustrates $\psi$ for MWTWs from a patient at different $\mathrm{HD}$ time points $(\mathrm{H} 1-\mathrm{H} 3)$ as well as the corresponding functions $\ln (e(i))$ for varying $i$. 

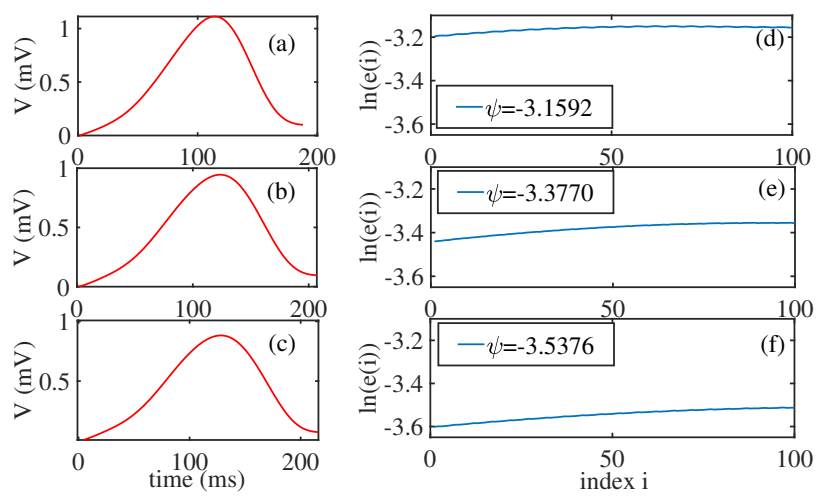

Figure 2. Panels a-c: MWTWs from a particular patient at different HD time points. Panels d-f: $\ln (e(i))$ versus index $i$ varying from 1 to $I$ as described in the text. $\psi$ values obtained as the average of $\ln (e(i))$ for all values of $i$ are shown for each MWTW.

In each patient and simulated fiber, $\left[\mathrm{K}^{+}\right]^{*}$ and $\psi^{*}$ were used to denote the values of $\left[\mathrm{K}^{+}\right]$and $\psi$ associated with minimum $\psi$. We denoted $\Delta\left[\mathrm{K}^{+}\right]=\left[\mathrm{K}^{+}\right]-\left[\mathrm{K}^{+}\right]^{*}$ and $\Delta \psi=\psi-\psi^{*}$. Pearson correlation coefficients were computed to assess the strength of the linear relationship between $\psi$ and $\left[\mathrm{K}^{+}\right]$. Linear partial correlation coefficients were computed while controlling for the effects of $\left[\mathrm{Ca}^{2+}\right]$ and heart rate.

\subsection{Sensitivity Analysis}

To assess how transmural heterogeneities modulated $\psi$ at different $\left[\mathrm{K}^{+}\right]$concentrations, a sensitivity analysis was performed. The percentage of change and the sensitivity of $\psi\left(S_{\psi}\right)$ to variations in the proportion of endo-, mid- and epicardial cells were calculated as described in $[5,13]$.

\section{Results and Discussion}

Figure 3 shows the regression lines obtained from linearly fitting $\Delta \psi$ vs $\Delta\left[\mathrm{K}^{+}\right]$for each of the analyzed ESRD patients (left panel) and each of the simulated ventricular fibers (right panel). The range of $\left[\mathrm{K}^{+}\right]$values in the simulations corresponds to the range calculated from the patients' data. As can be observed from the figure, the simulated results reproduce the behavior observed from the clinical ECGs, including the high inter-individual variability. In the patients, $\Delta \psi$ took the largest values at the beginning of the HD session, corresponding to the highest $\left[\mathrm{K}^{+}\right]$values. Similarly, in the simulated fibers, the highest $\Delta \psi$ values were found under hyperkalemia.

Results presented in Figure 4 illustrate the strong linear correlation between $\Delta \psi$ and $\Delta\left[\mathrm{K}^{+}\right]$in the patients ( $\rho_{A}$, with a median value of 0.92 ) and in the simulated fibers $\left(\rho_{C}\right.$, median of 0.84$)$. Also, the figure shows that,
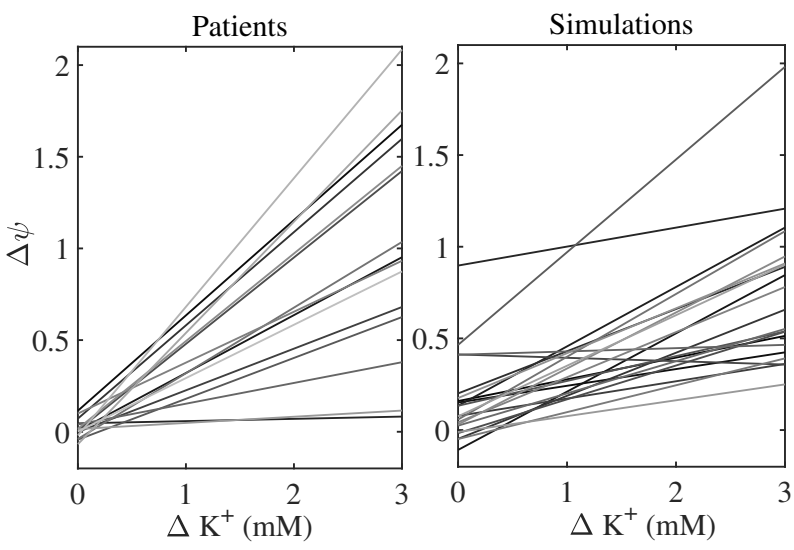

Figure 3. Fitted linear regression models to the relationships between $\Delta \psi$ and $\Delta\left[\mathrm{K}^{+}\right]$for the analyzed patients (left panel) and simulated fibers (right panel).

even after controlling for additional effects, the partial correlation between $\psi$ and $\left[\mathrm{K}^{+}\right]$in the patients is still high: median $\rho_{B}$ of 0.74 after accounting for both heart rate and $\left[\mathrm{Ca}^{2+}\right]$ variations.

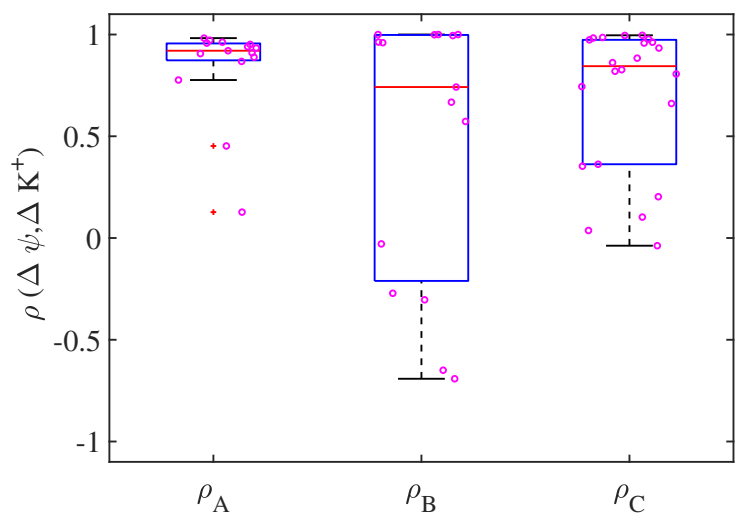

Figure 4. Boxplots of Pearson correlation coefficients between $\Delta \psi$ and $\Delta\left[\mathrm{K}^{+}\right]$for the analyzed patients $\left(\rho_{A}\right)$ and simulated fibers $\left(\rho_{C}\right)$ as well as boxplot of linear partial correlation coefficients for the patients after controlling for the effects of both $\left[\mathrm{Ca}^{2+}\right]$ and heart rate $\left(\rho_{B}\right)$. Each dot represents a patient or a simulated fiber.

Figure 5 shows the results from the sensitivity analysis for $\psi$. As can be observed, the largest sensitivity of $\psi$ was found to variations in the proportion of epi- and midmyocardial cells within the ventricular wall. This was particularly more prominent when $\left[\mathrm{K}^{+}\right]$became elevated above physiological levels. For $\left[\mathrm{K}^{+}\right]=7 \mathrm{mM}$, sensitivity values above $100 \%$ and $80 \%$ were found for epiand midmyocardial proportions, which means that $10 \%$ variations in the epi- and midmyocardial proportions led 
to changes larger than $10 \%$ and $8 \%$ in $\psi$.

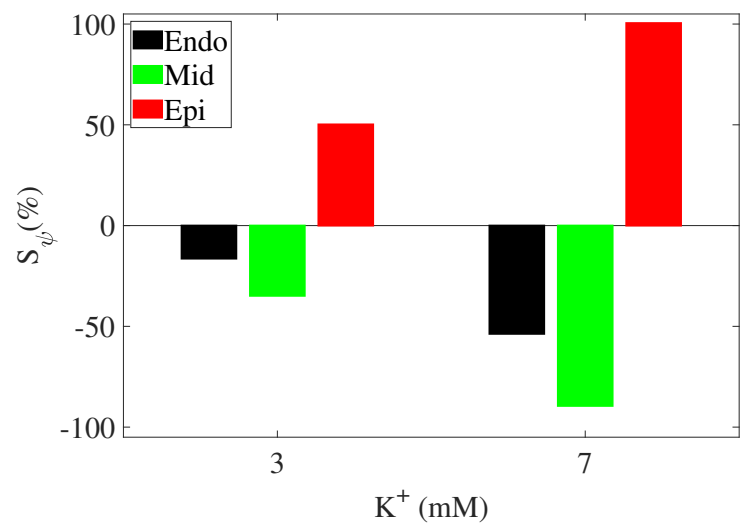

Figure 5. Sensitivity of $\psi$ to variations in the proportion of endo-, mid- and epicardial layers of the simulated ventricular fibers at two different $\left[\mathrm{K}^{+}\right]$levels.

\section{Conclusions}

The divergence-related marker $\psi$, as a descriptor of the nonlinear dynamics of the $\mathrm{T}$ wave, presents remarkable changes with varying $\left[\mathrm{K}^{+}\right]$in ESRD patients, although high inter-individual variability in the $\psi$ - $\left[\mathrm{K}^{+}\right]$relationship is observed. The wide range of strengths and patterns of such relationship in the patients are well reproduced in silico. Transmural heterogeneities in simulated human ventricular fibers are shown to play a relevant role in determining the patient-specific response of the $\mathrm{T}$ wave to hypo/hyperkalemia.

\section{Acknowledgements}

This work was supported by projects ERC-StG 638284 (ERC), PID2019-105674RB-I00 and PID2019-104881RB-I00 (Ministerio de Ciencia e Innovación), Marie Skłodowska-Curie grant 764738 (European Commission) and by European Social Fund (EU) and Aragón Government through BSICoS group T39_20R and project LMP124-18. Computations were performed by the ICTS NANBIOSIS (HPC Unit at University of Zaragoza).

\section{References}

[1] Hill NR, et al. Global prevalence of chronic kidney disease - A systematic review and meta-analysis. PloS One 2016; 11(7):e0158765.

[2] Weiss JN, et al. Electrophysiology of hypokalemia and hyperkalemia. Circulation Arrhythmia and Electrophysiology 2017;10(3).

[3] Corsi C, et al. Noninvasive quantification of blood potassium concentration from ECG in hemodialysis patients. Scientific Reports 2017;7:42492.

[4] Bukhari HA, et al. Transmural ventricular heterogeneities play a major role in determining T-wave morphology at different extracellular potassium levels. In 2019 Computing in Cardiology (CinC). September 2019; 1-4.

[5] Bukhari HA, et al. Characterization of T wave amplitude, duration and morphology changes during hemodialysis: Relationship with serum electrolyte levels and heart rate. IEEE Transactions on Biomedical Engineering 2020, Under review;.

[6] Martínez JP, et al. A wavelet-based ECG delineator: evaluation on standard databases. IEEE Transactions on Biomedical Engineering April 2004;51(4):570-581.

[7] Ramírez J, et al. Variability of ventricular repolarization dispersion quantified by Time-warping the morphology of the T-waves. IEEE Transactions on Biomedical Engineering 2017;64(7):1619-1630.

[8] Ten Tusscher KHWJ, et al. Alternans and spiral breakup in a human ventricular tissue model. American Journal of Physiology Heart and Circulatory Physiology September 2006;291(3):H1088-H1100.

[9] Severi S, et al. From in vivo plasma composition to in vitro cardiac electrophysiology and in silico virtual heart: the extracellular calcium enigma. Philosophical Transactions Series A Mathematical Physical and Engineering Sciences June 2009;367(1896):2203-2223.

[10] Heidenreich EA, et al. Adaptive macro finite elements for the numerical solution of monodomain equations in cardiac electrophysiology. Annals of Biomedical Engineering July 2010;38(7):2331-2345.

[11] Gima K, et al. Ionic current basis of electrocardiographic waveforms: a model study. Circulation Research May 2002;90(8):889-896.

[12] Rosenstein MT, et al. A practical method for calculating largest Lyapunov exponents from small data sets. Physica D Nonlinear Phenomena May 1993;65(1):117-134.

[13] Romero L, et al. Impact of ionic current variability on human ventricular cellular electrophysiology. American Journal of Physiology Heart and Circulatory Physiology October 2009;297(4):H1436-1445.

Address for correspondence:

Hassaan A. Bukhari; Institute of Engineering Research of Aragón, Universidad de Zaragoza (Spain).

hassaanahmed01@unizar.es 